\title{
Community and Individual level Factors Associated with Anemia among Lactating mothers in Ethiopia using data from Ethiopian Demographic and Health Survey, 2016; Multilevel analysis
}

Alemneh mekuriaw liyew ( $\square$ alemnehmekuriawliyew@gmail.com )

University of Gondar https://orcid.org/0000-0001-9034-6205

Achamyeleh Birhanu Teshale

University of Gondar

Research article

Keywords: Anemia Lactation multilevel analysis, Ethiopia

Posted Date: March 29th, 2020

DOl: https://doi.org/10.21203/rs.3.rs-18828/v1

License: (c) (1) This work is licensed under a Creative Commons Attribution 4.0 International License.

Read Full License

Version of Record: A version of this preprint was published at BMC Public Health on May 24th, 2020. See the published version at https://doi.org/10.1186/s12889-020-08934-9. 


\section{Abstract}

Introduction: Maternal anemia is a worldwide public health problem especially in developing countries including Ethiopia. The burden of anemia among lactating mothers in Ethiopia was higher than those who are neither pregnant nor breastfeeding. To date, there is limited evidence on community level determinates of anemia among lactating mothers in Ethiopia. Therefore, this study aimed to assess the individual and community level determinants of anemia among lactating mothers in Ethiopia.

Methods: Secondary data analysis was employed using 2016 Ethiopian demographic and health survey. A total of 4658 (weighted samples) breastfeeding women were included in the current study. Multilevel logistic regression model was used to identify individual and community level determinants of anemia during lactation. Finally, adjusted odds ratio with $95 \%$ confidence interval was reported.

Results: The overall prevalence of anemia was $28.3 \%(95 \% \mathrm{Cl} ; 26.7,30.0)$. With the higher regional prevalence in Somali (68\%) and Afar (47\%) regions. using modern contraceptive method [AOR $=0.71 ; 95 \%$ Cl: 0.58, 0.87]; Poorer [AOR $0.77 ; 95 \% \mathrm{Cl}: 0.61,0.98]$, middle $[\mathrm{AOR}=0.74 ; 95 \% \mathrm{Cl}: 0.56,0.97]$ rich $[\mathrm{AOR}=$ $0.64 ; 95 \% \mathrm{Cl}: 0.46,0.85]$, richest $[\mathrm{AOR}=0.66 ; 95 \% \mathrm{Cl}: 0.43,0.98]$ wealth index; working within the 12 months preceding the survey $[\mathrm{AOR}=0.77 ; 95 \% \mathrm{Cl}$ : 0.64, 0.92]; and taking iron during pregnancy $[\mathrm{AOR}=$ $0.82 ; 95 \% \mathrm{Cl}: 0.68,0.98]$ were associated with lower odds of anemia. Whereas, female household head $[A O R=1.22 ; 95 \% \mathrm{Cl}: 1.01,1.49]$ having two births $[A O R=1.27 ; 95 \% \mathrm{Cl}: 1.04,1.55]$, three to four births $[A O R=1.53 ; 95 \% \mathrm{Cl}: 1.14,2.06]$ and higher community illiteracy $[\mathrm{AOR}=1.06 ; 95 \% \mathrm{Cl}: 1.06,1.70]$ were associated with the increased odds of anemia during lactation.

Conclusion: In this study the prevalence of anemia among lactating mothers was high. It was affected by both community and individual-level factors. Therefore focusing on family planning services especially on modern contraceptive methods, child spacing and improving community literacy will help to reduce anemia during lactation.

\section{Introduction}

Anemia refers to a low hemoglobin level with a cutoff point $<110 \mathrm{~g} / \mathrm{L}$ for pregnant women and $<120 \mathrm{~g} / \mathrm{L}$ for non-pregnant women(1). Itis a disease which is characterized by a decreased number of red blood cells or hemoglobin level that results in insufficient oxygen-carrying capacity of blood to meet the cellular metabolic demand of the body.Nutritional deficiencies were the most common causes of anemia. Of these, Iron deficiency is the major contributor of anemia globally. However, folic acid, vitamin B12, and vitamin A deficiency also cause nutritional deficiency anemia. Other causes of anemia can be categorized as acute and chronic inflammations, parasitic infections, and acquired or inherited disorders that affect the synthesis of hemoglobin and production or survival of red blood cells $(2,3)$.

Anemia is a worldwide public health problem affecting both developing and developed countries which occurs in all population groups of the human being (2). The major consequences of anemia were increased mortality and morbidity for human health as well as poor social and economic development(2). 
World Health Organization (WHO)defines anemia as a major public health problem, moderate public health problem, and mild public health problem when prevalence is over $40 \%$, between 20 and $40 \%$ and between 5 and $20 \%$ respectively $(2,3)$.

Globally, 38\% of pregnant women and29\% of non-pregnant women were anemic in 2011.Pregnant women in low-income and middle-income countries (LMICs)had high rates of anemia, in which the highest prevalence rates are reported in Central and West Africa (56\%), SouthAsia (52\%) and East Africa (36\%) (4).Similarly, a large proportion of non-pregnant women were reportedly anemic in West and Central Africa (48\%), South Asia (47\%) and East Africa (28\%) (4).

In sub-Saharan Africa and South Asia, the evidence from a meta-analysis of observational and intervention trials showed that $\sim 20 \%$ of maternal mortality was attributed to anemia which was primarily the result of iron deficiency(5). Furthermore, anemia could have various adverse effects on a woman's health like maternal death and devastating morbidity $(6)$, depression $(7,8)$, raised blood pressure $(9,10)$, as well as adverse birth outcomes such as low birth weight and preterm birth (11). This, makes anemia to be one of the global health priority areas at the global level, especially in resource-limited areas (12). Therefore reducing anemia is considered as an essential part of improving the health of women, and the WHO has set a global target of achieving a $50 \%$ reduction of anemia among women of reproductive age by 2025 (13).

Postpartum anemia is highest in mothers who were anemic during pregnancy (14). Lactating mothers are vulnerable to anemia morbidity. This is because, during the period of lactation, mothers are susceptible to iron depletion and bad consequences of blood loss during childbirth(15). The concentration of iron in breast milk is independent of maternal iron status. This indicates that the quality of breast milk is maintained at the expense of maternal stores $(15,16)$. So far community-level factors that might affect anemia during lactation were largely overlooked. Therefore this study was aimed to identify the individual and community level determinants of anemia during lactation.

\section{Methods}

\section{Study design and setting}

Secondary data analysis was employed using the 2016 Ethiopian demographic and health survey.The study is conducted in Ethiopia ( $30-140 \mathrm{~N}$ and $330-48^{\circ} \mathrm{E}$ ), located at the horn of Africa (Fig. 1). The country covers 1.1 million Sq. $\mathrm{km}$ and has a great geographical diversity, which ranges $4550 \mathrm{~m}$ above sea level down to the Afar depression to $110 \mathrm{~m}$ below sea level. There are nine regional states and two city administrations subdivided into 68 zones, 817 districts and 16,253 kebeles (lowest local administrative units of the country) in the administrative structure of the country (17).

\section{Data source and measurements}


Every five years, the Demographic and Health Survey program of the country (EDHS) has collected data on national representative samples of all age groups and key indicators including anemia among reproductive-age women. The sociodemographic, socioeconomic, child health and maternal related variables were included in the questionnaire.

A stratified two-stage cluster sampling procedure was employed to select study participants. In 2016 survey 645 EAs (202 urban and 443 rural) were selected. From these enumeration areas, 18008 households and 16583 eligible women were selected. The hemoglobin level was measured for those eligible mothers after having consent and it was adjusted for altitude (17). In the current study, 4657 lactating mothers breastfeeding.

\section{Dependent variable}

The hemoglobin level was measured for those eligible mothers after having consent and it was adjusted for altitude. Therefore, the current study was based on the altitude adjusted hemoglobin level which was already provided in the EDHS data. Lactating mothers were considered to be anemic if their hemoglobin level was $<12 \mathrm{~g} / \mathrm{dL}$. Hemoglobin level was measured in $\mathrm{g} / \mathrm{dL}$, operationalized as a categorical variable by predefined cut-off points for mild, moderate and severe anemia recommended by the WHO for women above the age of 15 years. For this analysis, we recategorized anemia level as anemic and non-anemic from prior classifications in levels (no, mild, moderate, severe) because of very small numbers of cases in the categories of severe and mild anemia, Therefore, women with hemoglobin level $<120 \mathrm{~g} / \mathrm{L}$ were considered as anemic and coded as " 1 " whereas those nonanemic were coded as " 0 " for further analysis.

\section{Independent variables:}

From the 2016 EDHS datasets, the mothers' age, educational status of mother and husband, parity, wealth status, sex of household head, maternal BMI, ANC visit, cesarean delivery, history of a terminated pregnancy, smoking, health insurance, maternal occupation, religion, marital status, perception of distance from the health facility, source of drinking water, type of toilet facility, place of delivery, iron supplementation, use of current contraceptive, duration of breastfeeding, births in the past five years and birth interval were considered as individual-level variables.

Whereas community poverty, community media exposure, community illiteracy level and place of residence were community-level variables. The aggregate community level explanatory variables were constructed by aggregating individual-level characteristics at the community (cluster) level. They were dichotomized as high or low based on the distribution of the proportion values computed for each community after checking the distribution by using the histogram. If the aggregate variable was normally distributed mean value and if not, normally distributed median value was used as a cut-off point for the categorization. Community poverty level was categorized as high if the proportion of women from the two lowest wealth quintiles in a given community was $50-100 \%$ and low if the proportion was $0-49 \%$. 
Community media exposure was categorized as low if the proportion of women exposed to media in the community was $0-28.60 \%$ and categorized as high if the proportion was $27-100 \%$. Community illiteracy level was categorized as high if the proportion of illiterate women per cluster was $83.3-100 \%$ and low if it was less than $83.30 \%$.

\section{Model building}

Four models were fitted. The first was the null model containing no exposure variables which was used to check variation in community and provide evidence to assess random effects at the community level. The second model was the multivariable model adjustment for individual-level variables and model three was adjusted for community-level factors. In the fourth model both individual and community level variables were fitted with the outcome variable.

\section{Parameter estimation method}

The fixed effects (a measure of association) were used to estimate the association between the likelihood of anemia and explanatory variables at both community and individual levels and were expressed as odds ratios with $95 \%$ confidence interval. Regarding the measures of variation (randomeffects) intracluster correlation coefficient (ICC), Proportional Change in Community Variance (PCV) and median odds ratio (MOR) were used.

The aim of the median odds ratio (MOR) is to translate the area level variance in the widely used odds ratio (OR) scale, which has a consistent and intuitive interpretation. The MOR is defined as the median value of the odds ratio between the area at the highest risk and the area at the lowest risk when randomly picking out two areas. The MOR can be conceptualized as the increased risk that (in median) would have if moving to another area with a higher risk.

It is computed by; MOR=exp[ $\sqrt{ }(2 \times V a) \times 0.6745]$

Where; VA is the area level variance, and 0.6745 is the 75 th centile of the cumulative distribution function of the normal distribution with mean 0 and variance 1 . See elsewhere for a more detailed explanation (18). Whereas the proportional change in variance is calculated as $\mathrm{PCV}=[(\mathrm{VA}-\mathrm{VB}) /$ $\mathrm{VA}] * 100$;

Where; where $\mathrm{VA}=$ variance of the initial model, and $\mathrm{VB}=\mathrm{variance}$ of the model with more terms

\section{Ethical consideration}


Ethical clearance was approved by an Institutional ethical Review committee of the Institute of Public Health, College of Medicine and Health Sciences, University of Gondar. The approval letter for the use of the EDHS data set was also gained from the Measure DHS (ORC MACRO). No information obtained from the data set was disclosed to any third person.

\section{Results}

\section{Sociodemographic characteristics of study participants}

In this study, we used weighted samples of 4658 breastfeeding women. The median age of the study participants was 28 (IQR=24-33) years. About half (49.52\%) of study subjects had no formal education and majority $(39.17 \%)$ of respondents were followers of orthodox religion. Regarding household status, $22.64 \%$ and $22.63 \%$ of study subjects were from poorest and poorer households. The majority $(74.70)$ of lactating mothers had normal nutritional status (BMI=18.5-24.99) and $95.21 \%$ of respondents were not covered by health insurance. Most (72.29\%) of respondents had no media exposure and $59.29 \%$ perceived distance from the health facility as a big problem. Looking to source of drinking water and type of toilet facility, $42.97 \%$ and $91.21 \%$ of study subjects used unimproved water sources for drinking and unimproved toilet facility respectively. Regarding respondent's residence and region, $89.26 \%$ and $96.65 \%$ of respondents were from rural residence and agrarian regions respectively (Table 1).

Table 1: Sociodemographic characteristics of lactating mothers in Ethiopia, 2016 


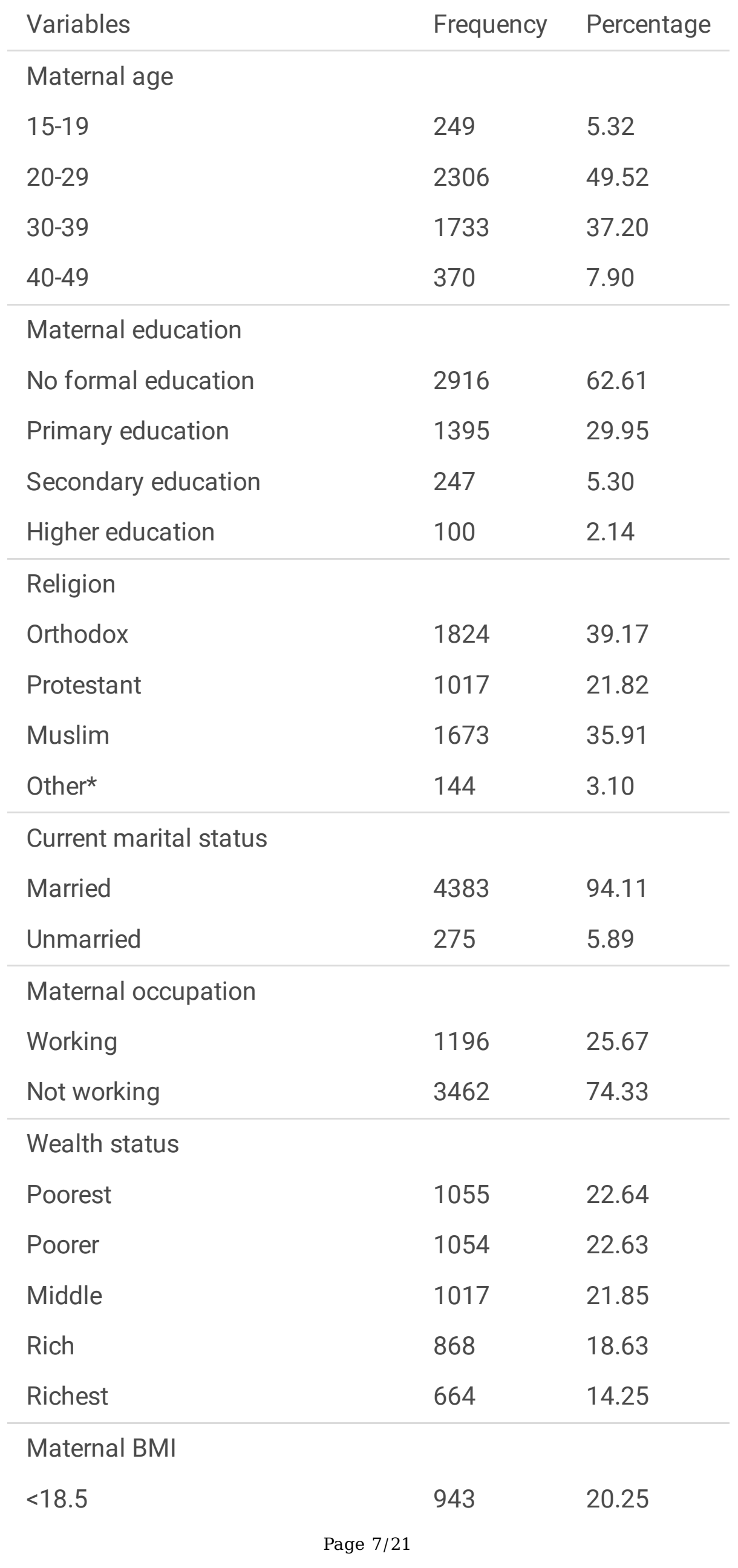




\begin{tabular}{|c|c|c|}
\hline $18.5-24.99$ & 3479 & 74.70 \\
\hline$>=25$ & 263 & 5.05 \\
\hline \multicolumn{3}{|l|}{ Smoking } \\
\hline Yes & 32 & 0.68 \\
\hline No & 4626 & 99.32 \\
\hline \multicolumn{3}{|c|}{ Health insurance coverage } \\
\hline Yes & 224 & 479 \\
\hline No & 4434 & 95.21 \\
\hline \multicolumn{3}{|c|}{ distance from the health facility } \\
\hline \multicolumn{3}{|l|}{ Big problem } \\
\hline \multirow[t]{2}{*}{ Not big problem } & 2762 & 59.29 \\
\hline & 1896 & 40.71 \\
\hline \multicolumn{3}{|l|}{ Media exposure } \\
\hline Yes & 1291 & 27.71 \\
\hline No & 3367 & 72.29 \\
\hline \multicolumn{3}{|c|}{ Source of drinking water } \\
\hline Pipe & 1242 & 26.66 \\
\hline Other improved & 1415 & 30.37 \\
\hline Not improved & 2001 & 42.97 \\
\hline \multicolumn{3}{|c|}{ Type of toilet facility } \\
\hline Improved & 405 & 8.69 \\
\hline Not improved & 4253 & 91.31 \\
\hline \multicolumn{3}{|l|}{ Place of delivery } \\
\hline Home & 3104 & 66.65 \\
\hline Health facility & 1554 & 33.35 \\
\hline \multicolumn{3}{|l|}{ Delivery by CS } \\
\hline Yes & 115 & 2.46 \\
\hline No & 4543 & 97.54 \\
\hline
\end{tabular}




\begin{tabular}{|c|c|c|}
\hline \multirow[t]{2}{*}{ No } & 382 & 8.19 \\
\hline & 4276 & 91.81 \\
\hline \multicolumn{3}{|l|}{ ANC visit } \\
\hline No visit & 1592 & 34.18 \\
\hline one-three & 1513 & 32.48 \\
\hline four and above & 1553 & 33.34 \\
\hline \multicolumn{3}{|c|}{ Iron supplementation } \\
\hline \multicolumn{3}{|l|}{ No } \\
\hline \multirow[t]{2}{*}{ Yes } & 2482 & 53.28 \\
\hline & 2176 & 46.72 \\
\hline \multicolumn{3}{|c|}{ modern contraceptive use } \\
\hline \multicolumn{3}{|l|}{ Yes } \\
\hline \multirow[t]{2}{*}{ No } & 1615 & 65.34 \\
\hline & 3043 & 34.66 \\
\hline \multicolumn{3}{|c|}{ Duration of breast feeding } \\
\hline 12 months & 2242 & 48.13 \\
\hline 13 to 36 & 1483 & 31.84 \\
\hline 37 and above & 933 & 20.03 \\
\hline \multicolumn{3}{|c|}{ Births in the five years } \\
\hline One & 2461 & 52.84 \\
\hline Two & 1854 & 39.80 \\
\hline Three to four & 343 & 7.37 \\
\hline \multicolumn{3}{|l|}{ Parity } \\
\hline Primiparous & 919 & 19.71 \\
\hline Multiparous & 1935 & 41.53 \\
\hline Grand multiparous & 1804 & 38.75 \\
\hline \multicolumn{3}{|l|}{ Birth interval } \\
\hline$<24$ month & 579 & 15.47 \\
\hline$>=24$ month & 3159 & 84.53 \\
\hline
\end{tabular}


Urban

501

10.74

Rural

4157

89.26

Community level media exposure

Low

High

2130

45.72

2528

54.28

Community illiteracy level

Low

2444

52.48

High

2214

47.52

Community poverty level

Low

2818

60.49

High

1839

39.51

Note; Other*=catholic, traditional \& other

\section{Prevalence of anemia among lactating mothers in Ethiopia, 2016}

The prevalence of anemia in this study was $28.3 \%(95 \% \mathrm{Cl} ; 26.7,30.0)$. Regarding the regional prevalence of anemia during lactation, the highest prevalence was observed in Somali region ( $68.31 \%)$ followed by Afar region. (Figure 1).

Fig.1.prevalence of anemia among lactating mothers across regions in Ethiopia 2016.

\section{Random effect and model comparison}

Table 3 revealed the random effect or community variation and model comparison. As indicated from the table, the ICC in the null model was 0.21 , which means about $21 \%$ of the variations of anemia in lactating mothers were attributable to the difference at cluster level or community-level factors. The higher MOR value (2.46) in the null model also revealed that anemia among lactating mothers were different between clusters or enumeration areas. Furthermore, the higher PCV value (44\%) in the final model indicates that about $44 \%$ of the variation of anemia among lactating mothers was attributable to both the individual level and community-level factors. Regarding model comparison, we used deviance and the model with lowest deviance value (Model IV) was the best-fitted model. 
Table 2: Random effect and model comparison for factors associated with anemia among lactating mothers in Ethiopia, 2016

\begin{tabular}{|c|c|c|c|c|}
\hline Parameter & model I & Model II & Model III & Model IV \\
\hline ICC & $0.21(0.17-0.26)$ & $0.13(0.10-0.18)$ & $0.16(0.13-0.21)$ & $0.13(0.10-0.18)$ \\
\hline PCV & Reference & 0.43 & 0.27 & 0.44 \\
\hline MOR & $2.46(2.18-2.77)$ & $1.97(1.75-2.20)$ & $2.15(1.91-2.41)$ & $1.95(1.75-2.20)$ \\
\hline \multicolumn{5}{|c|}{ Model fitness } \\
\hline Deviance & 4895.418 & 4702.346 & 4787.795 & 4690.300 \\
\hline
\end{tabular}

\section{Determinants of anemia among lactating mothers}

In the bivariable multilevel logistic regression analysis all factors, except maternal age, smoking, marital status, birth interval, health insurance coverage, and religion, were associated with anemia in lactating mother $(p<0.20)$. In the multivariable analysis household wealth status, maternal working status within the 12 months preceding the survey, sex of household head, current modern contraceptive usage, iron supplementation during their last pregnancy, number of births within five years and community illiteracy level was significantly associated with anemia in lactating women $(p<0.05)$.

Mothers from poorer, middle, rich and richest households had 23\% [Adjusted odds ratio (AOR) $=0.77 ; 95 \%$ Cl: $0.61,0.98], 26 \%$ [AOR $=0.74 ; 95 \% \mathrm{Cl}: 0.56,0.97], 36 \%$ [AOR $=0.64 ; 95 \% \mathrm{Cl}: 0.46,0.85]$, and $34 \%$ [AOR $=$ $0.66 ; 95 \% \mathrm{Cl}: 0.43,0.98$ ] lower odds of having anemia as compared to mothers from poorest households. Looking at the sex of the household head, being mothers from households with female household head had 1.22 [AOR $=1.22 ; 95 \% \mathrm{Cl}: 1.01,1.49]$ times higher odds of having anemia. The odds of anemia was $23 \%$ [AOR $=0.77 ; 95 \% \mathrm{Cl}: 0.64,0.92]$ lower among lactating mothers who had been working within the 12 months preceding the survey as compared to their counterparts. The odd of having anemia was $18 \%$ $[A O R=0.82 ; 95 \% \mathrm{Cl}: 0.68,0.98]$ lower in mothers who took iron during their last pregnancy as compared to their counterpart. Lactating mothers who use modern contraceptive methods currently have $29 \%$ [AOR $=0.71 ; 95 \% \mathrm{Cl}: 0.58,0.87$ ] lower odds of having anemia. Regarding number of births within five years, lactating mothers who had two and three to four births had 1.27 [AOR $=1.27 ; 95 \% \mathrm{Cl}: 1.04,1.55]$ and 1.53 $[A O R=1.53 ; 95 \% \mathrm{Cl}: 1.14,2.06]$ times higher odds of anemia as compared to mothers who had one birth within five years. Moreover, lactating mothers from communities with higher illiteracy had 1.34 [AOR = $1.06 ; 95 \% \mathrm{Cl}: 1.06,1.70]$ times higher odds of anemia as compared to their counterparts (Table 2).

Table 3: multivariable multilevel analysis of factors associated with anemia among lactating mothers in Ethiopia, 2016 
Variables

Model Model II Model III Model IV

AOR $95 \% \mathrm{Cl} \quad$ AOR $95 \% \mathrm{Cl} \quad$ AOR $95 \% \mathrm{Cl}$

\section{Maternal education}

No formal education

1.00

1.00

Primary education

$0.96(0.79-$

$1.00(0.82-1.22)$

Secondary education

1.17)

$1.14(0.80-1.63)$

Higher education

1.07(0.76-

1.52)

$1.51(0.90-2.52)$

$1.41(0.85-$

2.35)

\section{Maternal occupation}

Working

0.76(0.63-

Not working

\section{Wealth status}

Poorest

Poorer

Middle

Rich

Richest
1.00

0.71(0.57-

$0.89)$

$0.64(0.50-$

$0.83)$

0.53(0.40-

$0.70)$

0.52(0.37-

0.74 )
1.00

0.77(0.61-0.98)

*.74(0.56-0.97)

0.64(0.46-0.85)

$0.66(0.43-0.98)$

\section{Sex of HH head}

Female

Male
1.23(1.02-

1.50)

1.00
1.22(1.01-1.49)

1.00

1.00

$1.2(1.00-$

1.43)

1.78(0.99-1.41)

0.82(0.60-1.13)

$0.84(0.61-$

1.15) 
Big problem

Not big problem

\section{Media exposure}

Yes

0.91(0.75-

1.11)

0.92(0.75-1.14)

No

1.00

1.00

\section{Source of drinking water}

Pipe

1.00

1.00

Other improved

1.18(0.93-

1.50)

1.18(.93-1.51)

Not improved

1.14(0.90-

1.45)

\section{Type of toilet facility}

Improved

1.00

1.00

Not improved

$0.80(0.61-$

1.04)

0.82(0.62-1.07)

\section{Place of delivery}

Home

1.00

1.00

Health facility

0.93(0.76-

1.14)

0.96(0.78-1.18)

\section{Delivery by CS}

Yes

$0.81(0.49-$

1.34)

$0.81(0.49-1.34)$

No

1.00

\section{Ever had of a terminated pregnancy}

Yes

No

$0.76(0.57-$

1.02)

1.00
$0.76(0.57-1.02)$

1.00

\section{ANC visit}

No visit

1.00

1.00

one-three

1.05(0.85-

1.30)

1.06(0.86-1.32) 
four and above

0.97(0.77-

1.23)

Iron supplementation during pregnancy

Yes

No

$0.81(0.68-$

0.97)

0.82(0.68-0.98)

1.00

1.00

\section{Current use of modern contraceptive}

Yes

No

$0.69(0.56-$

$0.84)$

$\underset{\star \star \star \star}{0.71}(0.58-0.87)$

1.00

1.00

Month on breast feeding

12 months

1.00

1.00

13 to 36

$0.88(0.74-$

1.05)

37 and above

$0.89(0.70$

1.12)

Births in the five years

One

1.00

1.00

Two

1.29(1.06-

Three to four

1.57)

1.27(1.04-1.55)

1.59(1.19-

2.14)

$1.53(1.14-2.06)$

Parity

Primiparous

1.00

1.00

Multiparous

1.07(0.83-

1.37)

1.08(0.84-1.39)

Grand multiparous

1.21(0.92-

1.23(0.94-1.62)

$1.60)$

\section{Residence}

Urban

1.00

1.00

Rural

$1.38(0.99$

1.91)

1.13(0.75-1.72) 
Low

High

$1.00 \quad 1.00$

0.91(0.73-

1.13)

\section{Community illiteracy level}

Low

1.00

1.00

High

$1.60(1.26-$

2.04)

$1.34(1.06-1.70)$

\section{Community poverty level}

Low

1.00

1.00

High

$1.65(1.28-$

2.12)

$1.15(0.88-1.51)$

Note; $\mathrm{AOR}=$ Adjusted Odds Ratio; $\mathrm{Cl}=$ Confidence Interval, $*=\mathrm{P}<0.05, * \star=\mathrm{P}<0.01$ and $* \star *=\mathrm{P} \leq 0.001$.

\section{Discussion}

Anemia in lactating mother is a neglected public health problem which has its own impact for both the mother and the newborn. Thus, we investigated the prevalence and determinants of anemia among lactating women in Ethiopia.

In this study, the prevalence of anemia among lactating mothers was $28.3 \%$ which was lower than studies in India (63\%), Vietnam (62\%), and Myanmar (60.3\%)(20-22) and higher than a previous study in Ethiopia using EDHS 2011 data which reported 22.1\% of lactating women had anemia (23). The current finding was similar to a study done in Jimma-Ethiopia (24), which showed a $28.7 \%$ prevalence of anemia among lactating mothers. The observed discrepancy (either lower or higher) between the other studies might be due to the difference in the study setting and study period. The other possible explanation might be due to the sociocultural difference between other countries and Ethiopia.

Consistent with a study in Ethiopia based on EDHS 2011 (23), we found that lactating mothers who had been working within the 12 months preceding the survey had lower odds of having anemia. This might be because mothers who were working can have a good income and buy the necessary and variety of foods for the benefit of her health and newborn's health. The other possible explanation is mothers who were working might have great confidence and decision-making power on their health.

Similarly, mothers who were from poorer, middle, rich and richest households had lower odds of having anemia as compared to those from lower households. This finding is supported by studies in Nepal (25), Myanmar (22), Rwanda (26), and Ethiopia (23). This might be because mothers from rich households had a great opportunity to have a balanced diet in terms of meal frequency and variety of food. This study also indicated that mothers who had supplemented with iron during their last pregnancy had lower 
odds of having anemia as compared to their counterparts. This is supported by a study in Bahir DarEthiopia (27), which showed that iron supplementation during pregnancy is negatively associated with having anemia both for pregnant and lactating women. The possible explanation could be, iron is the most important nutrient which is used for the formation of red blood cells and when it was taken during pregnancy it can have a probability of preventing anemia during the locational period as well.

Consistent with a study done in Nepal (25) and Ethiopia (28), which indicated being male household head lower the chances of the mothers to be anemic, in our study households who had female head had higher odds of anemia as compared with those households whose head was male. This may be due to the fact that awareness towards anemia and treatment-seeking behaviors for any health problems might be lower in female-headed households. This study also revealed that lactating mothers who were taking modern contraceptives had a lower risk of having anemia. This is congruent with studies done in low- and middleincome countries (29), sub-Saharan Africa (30), Rwanda (26) and Ethiopia (23). This is because taking contraceptives might have a chance of reduction of the monthly (menstrual) bleeding. Another possible explanation is that taking modern contraceptive decrease the risk of anemia due to hemorrhage during pregnancy and post-partum by reducing the number of pregnancy and childbirth since in this study had more than one birth within five years was a risk for acquiring anemia.

The number of births a woman had with five years is another factor associated with anemia among lactating mothers which revealed that mothers who had two and three to four children within five years had higher odds of having anemia. This finding is supported by a study in Ethiopia (31) which showed having too frequent birth is among a significant predictor of anemia. This is due to the fact that too many births in short period of time (within five years) might not give enough time to replenish or substitute lost nutrient stores before another reproductive cycle starts and which result iron deficiency anemia. Besides, mothers with frequent birth might have both antepartum and postpartum hemorrhage with subsequent births, which intern result chronic and repeated anemia.

Moreover, in our study higher community illiteracy level was another important factor which was associated with higher odds of having anemia in lactating mother. Another study also revealed that maternal health service utilization is associated with literacy level in the community in which mothers from communities with higher illiteracy level had higher odds of utilizing maternal health services (32). The possible reason for the association of women illiteracy level with anemia in lactating mothers might be lower education level decreases communication within the family particularly with the husband on health-related issues. In addition, illiteracy prevents women from developing confidence to make decisions regarding their health and seek out quality health services. In remote societies, higher proportions of illiterate females also indicate lower autonomy which intern result restriction from accessing important maternal health care services during pregnancy, childbirth and the postpartum period which finally end up with comorbidities like anemia.

The main strength of this study was the use of nationally representative data, based on laboratoryconfirmed anemia, which makes the findings of the study more representative. The other strength is the 
use of appropriate model (multilevel) for analyzing the data. But some important variables which are known to cause anemia such as helminthic infection and protozoan infections like malaria were not assessed. In addition, due to the cross-sectional nature of the data it is difficult to show cause and effect relationship between independent and dependent variables.

\section{Conclusion}

In this study the prevalence of anemia among lactating mothers was high. Both individual-level factors and community-level factors were associated with anemia among lactating mothers. Mothers from rich households, those who had been working in the 12 months preceding the survey, current modern contraceptive users, those mothers who had been taking iron during the last pregnancy, having more than one number of births within five years had lower odds of having anemia. While being mothers from households with female household heads and being from communities with higher proportions of illiterate women increases the odds of having anemia. Therefore, taking special attention to those highrisk groups could decrease anemia among lactating mothers.

\section{Declarations}

\section{Availability of Data and Materials}

All relevant data are available within the manuscript

\section{Ethical consideration}

Ethical clearance was approved by an Institutional ethical Review committee of the Institute of Public Health, College of Medicine and Health Sciences, University of Gondar. The approval letter for the use of the EDHS data set was also gained from the Measure DHS (ORC MACRO). No information obtained from the data set was disclosed to any third person.

\section{Computing interest}

Both authors declare that they have no competing interests.

Funding: The authors received no specific funding for this work.

\section{Acknowledgments}

The authors would like to thank measure DHS for their permission to access the DHS datasets and central statistical agency for the shapefile.

\section{Author's Contribution}


$A M L$ and $A B T$ involved in the design and conception of the study data analysis, interpretation and write up of the manuscript. Both the authors read and approved the manuscript.

\section{Abbreviations}

\begin{tabular}{ll} 
CSA & Central Statistical Agency \\
\hline EAs & Enumeration Areas \\
\hline EDHS & Ethiopia Demographic and Health Survey \\
\hline SNNP & South Nation Nationality and people \\
\hline WHO & World Health Organization
\end{tabular}

\section{References}

1. Geneva S. Haemoglobin Concentrations for the Diagnosis of Anaemia and Assessment of Severity. Vitamin and Mineral Nutrition Information System. Document Reference WHO. NMH/NHD/MNM/11.1. http://www. who. int/entity/vmnis/indicators/haemoglobin ..., 2011.

2. Organization WH. Assessing the iron status of populations: report of a joint World Health Organization/Centers for Disease Control and Prevention technical consultation on the assessment of iron status at the population level. : Geneva, Switzerland. . world health organization, 2007

3. De Benoist B, Cogswell M, Egli I, McLean E. Worldwide prevalence of anaemia 1993-2005; WHO Global Database of anaemia. 2008.

4. Stevens GA, Finucane MM, De-Regil LM, Paciorek CJ, Flaxman SR, Branca F, et al. Global, regional, and national trends in haemoglobin concentration and prevalence of total and severe anaemia in children and pregnant and non-pregnant women for 1995-2011: a systematic analysis of population-representative data. The Lancet Global Health. 2013;1(1):e16-e25.

5. Ross JS, Thomas EL. Iron deficiency anemia and maternal mortality. Working Notes Series. 1996;3.

6. Lumbiganon P, Laopaiboon M, Intarut N, Vogel J, Souza J, Gülmezoglu A, et al. Indirect causes of severe adverse maternal outcomes: a secondary analysis of the WHO Multicountry Survey on Maternal and Newborn Health. BJOG: An International Journal of Obstetrics \& Gynaecology. 2014;121:32-9.

7. Albacar G, Sans T, Martín-Santos R, García-Esteve L, Guillamat R, Sanjuan J, et al. An association between plasma ferritin concentrations measured $48 \mathrm{~h}$ after delivery and postpartum depression. Journal of affective disorders. 2011;131(1-3):136-42.

8. Corwin EJ, Murray-Kolb LE, Beard JL. Low hemoglobin level is a risk factor for postpartum depression. The Journal of nutrition. 2003;133(12):4139-42.

9. Aghamohammadi A, Zafari M, Tofighi M. High maternal hemoglobin concentration in first trimester as risk factor for pregnancy induced hypertension. Caspian journal of internal medicine. 
2011;2(1):194.

10. Zafar T, Iqbal Z. Iron status in preeclampsia. The Professional Medical Journal. 2008;15(01):74-80.

11. Rahman MM, Abe SK, Rahman MS, Kanda M, Narita S, Bilano V, et al. Maternal anemia and risk of adverse birth and health outcomes in low-and middle-income countries: systematic review and metaanalysis, 2. The American journal of clinical nutrition. 2016;103(2):495-504.

12. Kassebaum NJ, Jasrasaria R, Naghavi M, Wulf SK, Johns N, Lozano R, et al. A systematic analysis of global anemia burden from 1990 to 2010. Blood. 2014;123(5):615-24.

13. Targets WGN. 2025: anaemia policy brief. Geneva: World Health Organization. 2014.

14. Bodnar LM, Scanlon KS, Freedman DS, Siega-Riz AM, Cogswell ME. High prevalence of postpartum anemia among low-income women in the United States. American journal of obstetrics and gynecology. 2001;185(2):438-43.

15. Whitney E RS. . Understanding Nutrition. In: Adams P, ed. 11th edn. . USA: Thomson Learning Academic Resource Center; 2008:

16. Domellöf M, Lönnerdal B, Dewey KG, Cohen RJ, Hernell O. Iron, zinc, and copper concentrations in breast milk are independent of maternal mineral status. The American journal of clinical nutrition. 2004;79(1):111-5.

17. Central Statistical Agency (CSA) [Ethiopia] and ICF:2016. Ethiopia Demographic and Health Survey 2016 Addis Ababa, Ethiopia, and Rockville, Maryland, USA: CSA and ICF.

18. Goldstein H, Browne W, Rasbash J. Partitioning variation in multilevel models. Understanding Statistics: Statistical Issues in Psychology, Education, and the Social Sciences. 2002;1(4):223-31.

19. Merlo J, Yang M, Chaix B, Lynch J, Råstam L. A brief conceptual tutorial on multilevel analysis in social epidemiology: investigating contextual phenomena in different groups of people. Journal of Epidemiology \& Community Health. 2005;59(9):729-36.

20. Siddiqui MZ, Goli S, Reja T, Doshi R, Chakravorty S, Tiwari C, et al. Prevalence of anemia and its determinants among pregnant, lactating, and nonpregnant nonlactating women in India. Sage Open. 2017;7(3):2158244017725555.

21. Trinh LTT, Dibley M. Anaemia in pregnant, postpartum and non pregnant women in Lak district, Daklak province of Vietnam. Asia Pacific journal of clinical nutrition. 2007;16(2).

22. Zhao A, Zhang Y, Li B, Wang P, Li J, Xue Y, et al. Prevalence of anemia and its risk factors among lactating mothers in Myanmar. The American journal of tropical medicine and hygiene. 2014;90(5):963-7.

23. Lakew Y, Biadgilign S, Haile D. Anaemia prevalence and associated factors among lactating mothers in Ethiopia: evidence from the 2005 and 2011 demographic and health surveys. BMJ open. 2015;5(4):e006001.

24. Alemayehu M. Factors Associated with Anemia among Lactating Mothers in Subsistence Farming Households from Selected Districts of Jimma Zone, South Western Ethiopia: A Community Based 
Cross-Sectional Study. J Nutr Food Sci. 2017;7(595):2.

25. Nisar R, Anwar S, Nisar S. Food security as determinant of anemia at household level in Nepal. Journal of Food Security. 2013;1(2):27-9.

26. Habyarimana F, Zewotir T, Ramroop S. Spatial Distribution and Analysis of Risk Factors Associated with Anemia Among Women of Reproductive Age: Case of 2014 Rwanda Demographic and Health Survey Data. The Open Public Health Journal. 2018;11(1).

27. Feleke BE, Feleke TE. Pregnant mothers are more anemic than lactating mothers, a comparative cross-sectional study, Bahir Dar, Ethiopia. BMC hematology. 2018;18(1):2.

28. Ali JH. Gender differences in household headship and level of awareness on anaemia among Ethiopian women: Evidences from a nationwide cross-sectional survey. Ethiopian Journal of Health Development. 2018;32(2).

29. Bellizzi S, Ali MM. Effect of oral contraception on anemia in 12 low-and middle-income countries. Contraception. 2018;97(3):236-42.

30. Gebremedhin S, Asefa A. Association between type of contraceptive use and haemoglobin status among women of reproductive age in 24 sub-Saharan Africa countries. BMJ sexual \& reproductive health. 2019;45(1):54-60.

31. Alemu T, Umeta M. Reproductive and obstetric factors are key predictors of maternal anemia during pregnancy in Ethiopia: evidence from demographic and health survey (2011). Anemia. 2015;2015.

32. Singh PK, Kumar C, Rai RK, Singh L. Factors associated with maternal healthcare services utilization in nine high focus states in India: a multilevel analysis based on 14385 communities in 292 districts. Health policy and planning. 2014;29(5):542-59.

\section{Figures}




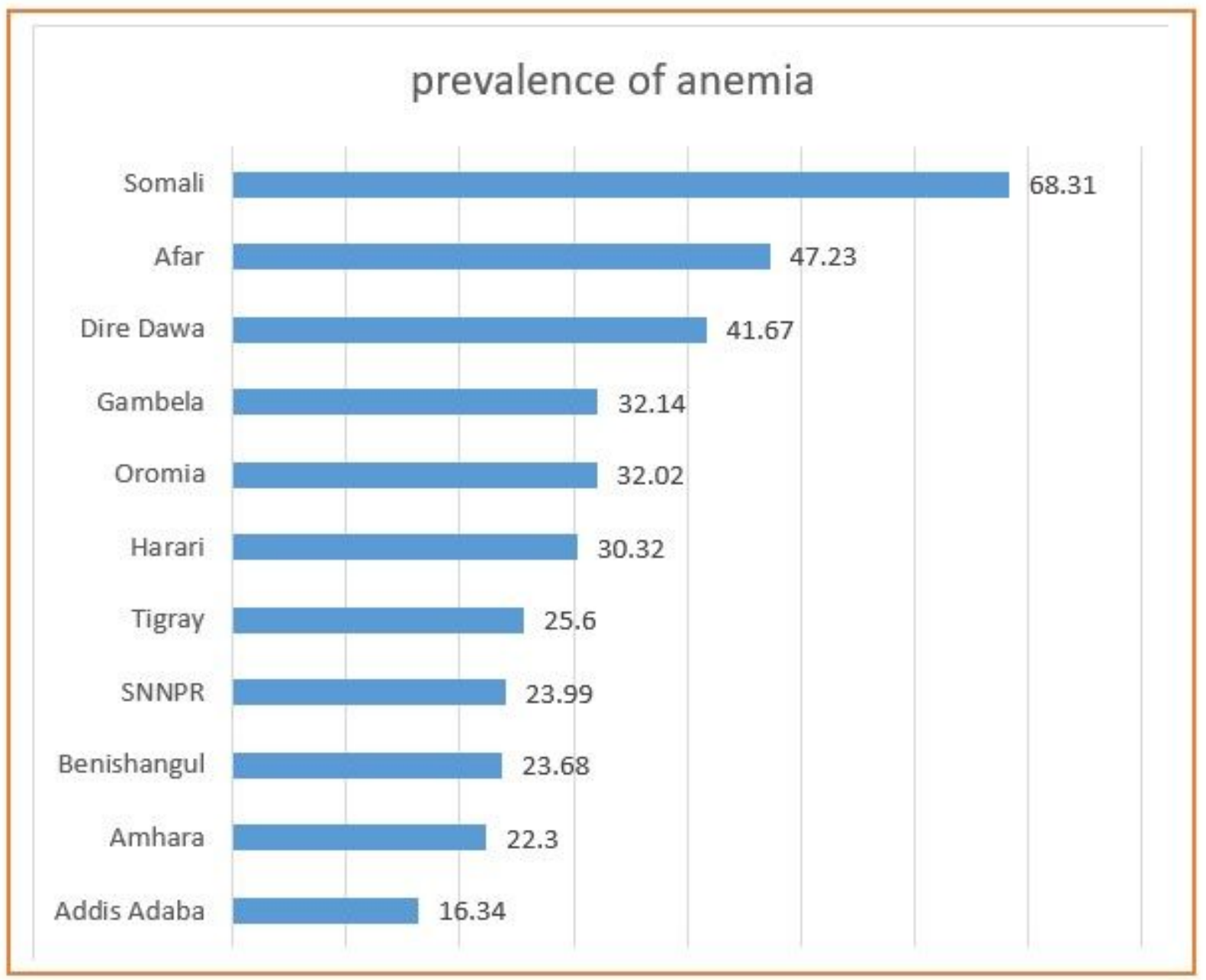

Figure 1

prevalence of anemia 